\title{
VULNERABILIDAD HUMANA EN TIEMPOS DEL POSHUMANO: UNA REFLEXIÓN TEOLÓGICA
}

\author{
LUIS MIGUEL TORRÓ FERRERO \\ Doctorando Teología Fundamental, \\ P.U. Gregoriana
}

\begin{abstract}
RESUMEN. El poshumanismo y transhumanismo representa varias y distintas corrientes de pensamiento que ponen de relieve la relación entre la tecnología y los seres humanos actualizando la pregunta acerca de qué es el ser humano. Algunas de estas propuestas sugieren que la tecnología será capaz de vencer todos los límites humanos, e incluso, los humanos serán capaces de conseguir la inmortalidad tecnológica. Pero, una mirada a lo que el ser humano es, indica la necesidad de comprenderlo como frágil y vulnerable. Existe un riesgo de deshumanización en la aplicación de las tecnologías si la vulnerabilidad humana no se tiene en cuenta.
\end{abstract}

PALABRAS CLAVE: poshumanismo, transhumanismo, antropología, tecnología, teología.

\section{Human Vulnerability in a Posthuman Age: a Theological Reflexion}

ABSTRACT. Posthumanism and Transhumanism are a set of several and distinct thinking trends that show the relation between technology and human beings updating the question about what the human being is. Some of these proposals suggest that the technology will be able to overcome all the human limits, and, even more, humans will be able to get a technological immortality. But, a look at what the human being is, points to the need of understanding it as fragile and vulnerable. There is a risk of dehumanization in applying technology if human vulnerability is not taken into consideration.

KEY WORDS: posthumanism, transhumanism, anthropology, technology, theology.

Es innegable que los tiempos en que nos ha tocado vivir están profundamente marcados por un intenso desarrollo científico-tecnológico. En este marco de desarrollo y transformación aparece el poshumanismo, una corriente de pensamiento muy amplia, compleja, multifacética e interdisciplinar que trata del ser humano en el encuentro con la tecnología y las ciencias ${ }^{1}$. El concepto es vago y ambiguo, como lo expresa Waters con rotundidad:

¿Qué es exactamente un poshumano? La pregunta es imposible de contestar definitivamente porque tal criatura no existe, y no hay un consenso entre los que especulan sobre su aparición. A pesar de la ambigüedad la perspectiva de un futuro poshumano genera una literatura creciente $^{2}$. De hecho, a pesar de las dificultades conceptuales, hay un creciente interés académico por la cuestión. Al hablar de

1 Cf. Farisco, M., «Posthuman Condition», en: Runehov, A., Oviedo, Ll., (eds.), Encyclopedia of Sciences and Religions, Springer Netherlands, Dordrecht, 2013, pp. 1815-1817.

2 La traducción es nuestra de: WATERs, B., From human to posthuman: Christian theology and technology in a postmodern world, Ashgate, Farnham, 2012, p. 50: «What exactly is a posthuman? The question is impossible to answer definitively because no such creature yet exists, and there is 
poshumanismo es importante notar que nos encontramos ante un conjunto muy amplío de corrientes diversas ${ }^{3}$, pero conviene, al menos, distinguir dos grandes bloques que, siguiendo a Tirosh-Samuelson ${ }^{4}$ podemos denominar: poshumanismo cultural o simplemente poshumanismo por una parte y poshumanismo tecnófilo o transhumanismo por otra.

Aún a riesgo de una excesiva generalización, diremos que en general el poshumanismo comparte una visión débil del concepto humano, que queda señalado como obsoleto, y es gracias a la aplicación de la tecnología que nos abrimos a la recepción de un ser nuevo el poshumano. Para el primer grupo (el poshumanismo cultural) el poshumano ya ha llegado, sin embargo, para el segundo (poshumanismo tecnófilo) el poshumano está por llegar.

Desde nuestra perspectiva teológica nos centraremos más en el segundo de estos grupos, y sobre todo en algunos aspectos antropológicos que surgen a partir de estas propuestas, en efecto, como señala Damour, el transhumanismo no es una corriente importante porque tenga muchos seguidores sino por la gravedad de las cuestiones que suscita en torno al ser humano ${ }^{5}$.

En el fondo, la pregunta poshumana sigue siendo la pregunta por el ser humano. Se evidencia que la pregunta del salmo sigue abierta: "¿qué es el hombre para que te acuerdes de él, el hijo de Adán para que de él te cuides?»(Sal 8, 5). El mismo hecho de una multiplicidad de visiones antropológicas, algunas de ellas difícilmente reconciliables, como dice Ladaria ${ }^{6}$, pone de relieve que el ser humano es un misterio, imagen del misterio de Dios. Un misterio que sólo se desvela en Cristo.

Uno de los riesgos que debemos evitar cuando nos acercamos a la cuestión del poshumanismo es el quedarnos en los límites tecnológicos o la factibilidad de las propuestas tecno-científicas. Es decir, nos arriesgamos a quedarnos fascinados por el impacto tecnológico y, a no prestar suficiente atención a las cuestiones esenciales, como la cuestión sobre la forma como se entiende el propio ser humano. Una de estas cuestiones esenciales es precisamente la vulnerabilidad humana.

La vulnerabilidad humana, propiamente hablando, no es una noción bíblica ni teológica, pero nos permite enlazar con muchas perspectivas filosóficas y teológicas, y también con una tradición cristiana amplia. Es, además, un concepto amplio y abierto, que también está generando un interés creciente en un ámbito interdisciplinar. La vulnerabilidad constituye uno de los cuatro principios de bioética y derecho que propone la Declaración de Barcelona de 19987 , y que se ha introducido

little consensus among those who speculate on its emergence. Despite the ambiguity the prospect of a posthuman future is generating a growing body of literature».

3 Cf., a título de ejemplo: FerRando, F., «Posthumanism, transhumanism, antihumanism, metahumanism, and new materialisms: differences and relations», en: Existenz 8, 2013, pp. 26-32.

4 Cf. Tirosh-Samuelson, H., «Transhumanism as a secularist faith», en: Zygon, 47, 2012, pp. 710-734.

5 Cf. Damour, F., La tentation transhumaniste, Salvator, Paris, 2015, p. 110.

6 Cf. Ladaria, L. F., El hombre en la creación, Biblioteca de Autores Cristianos, Madrid, 2012, p. 91.

7 La Declaración de Barcelona fue el resultado de un grupo de trabajo de académicos europeos durante 1995-98, financiado con fondos europeos y publicado en: RENDTORFF, J. D., KemP, P., Basic Ethical Principles in European Bioethics and Law, Center for Ethics and Law, Copenhagen y Barcelona, 2000. 
en el artículo 8 de la Declaración Universal sobre Bioética y Derechos Humanos ${ }^{8}$, aprobada en la 33 ${ }^{\mathrm{a}}$ sesión de la Conferencia General de la UNESCO en 2005.

Solbakk sugiere un comprensión doble de la vulnerabilidad: una concepción amplia frente a una concepción restrictiva o minimalista ${ }^{9}$. La segunda invitaría a fijarnos en la categoría de vulnerable en poblaciones o personas concretas, la primera, en cambio, tiende hacia una comprensión de la vulnerabilidad, como elemento constituyente de la condición humana. Ambas comprensiones resultan importantes desde una óptica cristiana.

Una concepción restrictiva de la vulnerabilidad nos invita a fijarnos en los sectores de la población más vulnerables, en principio todos estamos de acuerdo en la búsqueda de soluciones técnicas y tecnológicas para mejorar las condiciones de vida.

Jean Vanier, fundador del Arca, nos recuerda que conviene descubrir y valorar el lugar de los vulnerables en nuestra sociedad, pues es un camino para hacernos auténticamente más humanos. Él señala, desde su propia experiencia, como los más vulnerables encuentran dificultades para la integración en este mundo dominado por la tecnología, en concreto hace referencia a las personas con algún tipo de minusvalía mental, pero no sólo señala las dificultades sino que invita a una mirada más profunda descubriendo como en muchas ocasiones hay una compensación en otras facetas. Sugiere no sólo valorar esas otras dimensiones, sino dejarnos transformar por ellas, llegando a afirmar: «las personas débiles pueden tocar y transformar a las fuertes ${ }^{10}$, y añadiendo lo importante que sería redescubrir el papel que pueden aportar a favor de un auténtico crecimiento humano.

Pero, también es importante una mirada a la vulnerabilidad en su comprensión más amplia o fuerte, una concepción en palabras de Croti, en las que,

"la vulnerabilidad y la dependencia son cualidades originales del ser humano, no características de la falta ni expresión de un déficit funcional. Por lo tanto, la vulnerabilidad constituye una condición ontológica, es decir, pertenece al ser humano, porque es cualidad original que determina la "condición humana"»"1.

El poshumanismo no tiene en cuenta suficientemente esta realidad del ser humano, pues como nos recuerda Coeckelbergh,

«Algunos quieren dar un paso más adelante (o eso parece) y utilizar la tecnología no sólo para mejorar su condición o la de otros, sino para mejorar el ser humano, para hacernos mejores-que-humanos. Consideran que el ser humano

8 UNESCO, Declaración universal sobre Bioética y Derechos Humanos: UNESCO [accedido: 21.1.2016], http://portal.unesco.org/es/ev.php-URL_ID=31058\&URL_DO=DO_TOPIC\&URL_SECTION=201.html.

9 Cf. SolbakK, J. H., «Vulnerabilidad: ¿un principio fútil o útil en la ética de la asistencia sanitaria?», en: Redbioética/UNESCO 3, 2011, p. 92.

10 La traducción es nuestra de: VANIER, J., Toute personne est une histoire sacrée, Plon, Paris, 1994, p. 11: «des personnes faibles peuvent toucher et transformer les forts».

${ }_{11}$ La traducción es nuestra de: Свотті, M., La ferita di Medusa: riflessione pedagogica sulla vulnerabilità umana e sociale, Vita e Pensiero, Milano, 2009, p. 205: «la vulnerabilità e la dipendenza sono qualità originarie dell'essere umano, non caratteristiche proprie di una mancanza o espressione di un deficit di funzionamento. La vulnerabilità costituisce perciò una condizione ontologica, ossia propria di ogni essere umano perché qualità originaria che determina la "condizione umana"». 
es maleable y mejorable. Tienen la esperanza de que el mejoramiento humano [human enhancement] nos hará menos vulnerables. Algunos incluso desean convertirse en completamente invulnerables. Tal vez quieren que el ser humano sea inmortal. Sin embargo, ¿'es esto deseable? ¿Es posible?» ${ }^{12}$

Sobre la segunda pregunta que lanza, sobre la posibilidad de la invulnerabilidad, él la cuestiona abiertamente al proponer que en realidad, lo que la experiencia pone de relieve es que efectivamente hay tecnologías que reducen ciertos ámbitos de nuestra vulnerabilidad, pero señala igualmente que éstas medidas y estrategias acaban por crear «nuevos riesgos y nuevas vulnerabilidades, en realidad transforman la vulnerabilidad humana más que reducirla considerablemente» ${ }^{13}$.

Quizás la primera pregunta que formulaba es más interesante, ¿son deseables la aplicación de estas tecnologías? Entender la fragilidad y la vulnerabilidad humanas en perspectiva de límite a superar puede que nos impida ver el papel que en la conformación del propio ser humano tienen. El transhumanismo, como dice González Melado, «no se da cuenta de que en el hecho propio de la vulnerabilidad, de su limitación en el tiempo y en el espacio, es donde encuentra el hombre su propia grandeza» ${ }^{14}$.

Hablar de vulnerabilidad es una invitación a cómo entendemos que es el ser humano. Burgos, desde una perspectiva personalista, propone:

«Aunque la persona tiene una vida propia, personal e intransferible, no es un ser cerrado en sí mismo, sino abierto, que necesita trascenderse y salir de sí para desarrollarse con plenitud. Todos los hombres necesitan salir de sí y hacerse don para los otros si quieren realizarse y lo hacen fundamentalmente, a través de sus capacidades y facultades más elevadas y espirituales: la afectividad, la inteligencia y la libertad» $»^{15}$.

Estar abiertos, estar en relación con los demás nos hace necesariamente vulnerables, pero esta vulnerabilidad es la que nos permite encontrarnos con el otro, y la que en definitiva nos muestra la realidad del amor. Esto nos permitiría abrirnos a una teología de la ternura, como nos propone Gayol ${ }^{16}$.

Algunas propuestas poshumanistas proponen conseguir una invulnerabilidad por la aplicación de la tecnología. Estas propuestas, nos advierte Lambert ${ }^{17}$, son potencialmente deshumanizantes. Por tanto, una atenta mirada a la vulnerabilidad

12 La traducción es nuestra de: Coeckelbergh, M., Human being @ risk: enhancement, technology, and the evaluation of vulnerability transformations, Springer, Dordrecht, 2013, p. 4: «Some people want to go one step further (or so it seems) and use technology not only to improve their individual condition or that of particular others but to enhance the human, to make us better-thanhuman. They consider the human malleable and improvable. They hope that human enhancement will make us less vulnerable. Some of them even desire to become completely invulnerable. Maybe they want the human to be immortal. But is this desirable? Is it possible?»

13 La traducción es nuestra de: Coeckelbergh, o.c., p. 5: "new risks and vulnerabilities, thus transforming human vulnerability rather than substantially reducing it".

14 González Melado, F. J., «Transhumanismo (humanity+). La ideología que nos viene», en: Pax Emerita, 6, 2010, p. 228.

15 Burgos, J. M., Antropología: una guía para la existencia, Palabra, Madrid 2013, p. 46.

16 Cfr. GAYOL, N., "Una aproximación antropológica a la teología de la ternura», en URíBARRI Bilbao, G. (ed.), Teología y nueva evangelización, Desclée de Brouwer y Universidad Pontificia Comillas, Bilbao y Madrid, 2005.

17 Cf. Lambert, D., «Faut-il se libérer de la fragilité? Questions posées par une robotisation des activités humaines", en ARs, B, (ed.), Fragilité, dis-nous ta grandeur! Un maillon clé au sein d'une anthropologie postmoderne, Cerf, Paris, 2013, 110. 
humana nos ayuda a mejorar la comprensión sobre el ser humano, y a crecer siendo auténticamente más humanos.

Doctorando Teología Fundamental, PUG

Luis Miguel Torró Ferrero

luistorroferrero@gmail.com

[Artículo aprobado para publicación en diciembre de 2016] 